\title{
Bifurcation Analysis of a Model of Cancer
}

\author{
Abdo M. Al-Mahdi \\ Department of Mathematics and Computer Science \\ Faculty of Science, Ibb University, Yemen \\ Mustafa Q. Khirallah \\ Department of Mathematics and Computer Science \\ Faculty of Science, Ibb University, Yemen \\ Department of Mathematics, Faculty of Science and Arts \\ Najran University, Saudi Arabia
}

doi: 10.19044/esj.2016.v12n3p67 URL:http://dx.doi.org/10.19044/esj.2016.v12n3p67

\begin{abstract}
In this paper, we study the bifurcation of a cancer model with completely unknown parameters. The bifurcation analysis of the biologically feasible steady-states of this model will be discussed. It is proved that the system appears to exhibit many cases of bifurcation for some ranges of system parameters. Numerical analysis and extensive numerical examples of the bifurcation for some ranges were carried out for various system parameter values and different initial densities.
\end{abstract}

Keywords: Immunotherapy, cancer, biomathematics, bifurcation

\section{Introduction}

Mathematics has contributed in a small way to the understanding of cancer by analysis and simulation of cancer models directed at discovering new insights. This is well evidenced by the publication of a special issue of the journal, Discrete and Continuous Dynamical Systems Series B (Horn and Webb, 2004), titled "Mathematical Models in Cancer," which contains twenty one papers concerned with modeling various types and aspects of cancer (Takeuchi et al., 2007).

However, cancer is one of the major diseases that befalls humanity. Cancer arises from a mutation of genes that are responsible for the correct reproduction of cells (Rajib and Alam, 2012). The final result is an invasive malignancy - a tumor-which grows uncontrolled. Classic treatments include the surgical removal of the tumor, radiotherapy, immunotherapy, and also chemotherapy, which involves the infusion of drugs that inhibit or stop the growth of tumor cells (Pillis et al., 2006). 
Cancer is one of the greatest killers in the world today. Hence, the control of tumor growth requires special attention. The response of the tumor disease to treatment depends on many factors, including tumor severity and patient's immune response. The mathematical modeling of cancer selfremission and tumor has been approached by a number of researchers using a variety of models over the past decades.

Tumor cells are characterized by a vast number of genetic and epigenetic events leading to the appearance of specific antigens called neoantigens which trigger an antitumoral response by the immune system. The characteristic feature of cancer is its ability to metastatize, causing tissue disruptions. Thus, this further simulates the immune system. All these observations have led to the formulation of a hypothesis of the immune system that may eliminate tumors (Gohary and Alwasel, 2009).

Cancer is one of the most difficult diseases to treat clinically, and it is considered one of the main causes of death. It is the second most fatal disease after cardiovascular diseases. The World Health Organization (WHO) estimates that the annual cancer-induced mortality number exceeds six million people. Accordingly, the fight against cancer is of major public health interest. For this and other economy-related reasons, a great research effort is underway to understand the dynamics of cancer and to predict the impact of any changes on the system reactors. Hence, mathematical models are required to help design therapeutic strategies. In cancer modeling, we have to care about the scaling problem, where the class of equations used to describe the model are to be determined. Indeed, there are three natural scales, which are connected to the different stages of the disease and have to be identified (Rihan et al., 2012).

The first is the subcellular or molecular scale, where we focus on studying alterations in the genetic expressions of genes contained in the nucleus of a cell. Based on the findings of two journals of applied mathematics, we know that special signals are received by the receptors on the cell surface and transmitted to the cell nucleus. The second is the cellular scale, which is an intermediate level between the molecular and the macroscopic scale. The third is the macroscopic scale, where we deal with heterogeneous tissues. In the heterogeneous tissues, some of the layers constituting the tumor, like the external proliferating layer, the intermediate layer, and the inner zone with necrotic cells, may occur as islands. This leads to a tumor comprised of multiple regions of necrosis, engulfed by tumor cells in a quiescent or proliferative state (Bellomo et al., 2008). In the case of macroscopic scale, we focus on the interaction between the tumor cells and normal cells, e.g., immune cells and blood vessels in each of the three layers. For more details about the description of the scaling problem and the passage 
from one scale to another, we refer to Bellomo et al. (2008) and Chaplain (2006).

Vaccines for cancer represent an alternative approach to the use of standard drugs. Cancer vaccines work differently from traditional vaccines, which prevent diseases by instructing the immune system on how to recognize and destroy a particular pathogen, in that they enlist the patient's immune system to destroy existing cancer cells. While simple in concept, the development of products has proven difficult. Therefore, the problems specially lie in eliciting sufficient, tumor-selective stimulation of an immune system that is already tolerant of cancer cells (Zou, 2005).

In the field of mathematical biology, it is possible to describe certain phenomena by mathematical models and derive knowledge from them. Specifically, the human immune system consists of detection systems and required weapons. These systems play important roles in defending against most pathogens. Cancer immunotherapy is the use of the immune system to reject cancer. The main premise is stimulating the patient's immune system to attack the malignant tumor cells that are responsible for the disease (Ghomanjani, 2012).

A cancer treatment by immunotherapy is important in biomathematics. Immunotherapy (also called biological therapy and biotherapy) uses the body's own immune system to fight cancer and to reduce treatment related side effects. One of the most challenging tasks in constructing a mathematical model of cancer treatment is the calculation of biological parameters from empirical data. This task becomes increasingly difficult if a model involves several cell populations and treatment modalities (Pillis et al., 2009).

The idea of manipulating immunity to cure cancer has been pursued in the laboratory, and has been clinically tested for decades (Zvia and Stanimir, 2012). Several papers have thoroughly examined mathematical models of immunotherapy interactions. As explained in Nani and Freedman (2000), the author presents a model of cancer treatment by immunotherapy that sees normal cells and cancer cells as competitors for common resources. In addition, the anti-cancer cells are regarded as predators to the cancer cells.

The paper has the following structure. In section 2 , the mathematical model and the corresponding governing equations are written. In section 3 , the equilibrium points with their eigenvalues are analyzed. In section 4 , the stability analyses of the equilibria $E_{1}, E_{2}$, and $E_{3}$ are studied, while the time series and face planes of the system behavior near some critical points with fixed values of parameters are done in section 5. In section 6, we give an analytical investigation of bifurcation cases and draw many bifurcation diagrams for those cases and others. Some numerical results are given in section 7. Finally, in section 8, conclusions from this work were drawn. 


\section{The Model}

Gohary and Alwasel (2009) developed a model for spontaneous tumor regression and progression which is an interaction between the anticancer agent cell, lymphocytes, and macrophages, which are natural killer cells that destroy malignant cells. Furthermore, they constructed the spontaneous tumor regression and progression system as a prey-predator like system.

From the resource, the following assumptions were considered throughout (Gohary and Alwasel, 2009):

1. The predators are T-lymphocytes and cytotoxic macrophages/natural killer cells of immune system, which attack, destroy, or ingest tumor cells.

2. The preys are tumor cells which are attacked and destroyed by immune cells. The predator has two states, hunting and resting, and destroying the prey. The tumor cells are caught by macrophages which can be found in all tissues in the body, and these tumor cells circulate in the blood system.

3. Macrophages absorb tumor cells, eat them, and release a series of cytokines which activate the resting T-lymphocytes that coordinate the counter attack.

4. The resting predator cells can also be directly stimulated to interact with antigens. These resting cells cannot kill tumor cells, but they are converted to a special type of T-lymphocyte cells called natural killer or hunting cells. In addition, they begin to multiply and release other cytokines that, moreover, stimulate more resting cells.

5. This conversion between hunting and resting cells result in a degradation of the resting cells undergoing natural growth and an activation of hunting cells.

6. To introduce the mathematical model, we assume that tumor cells are being destroyed at a rate proportional to the tumor cells densities according to the law of mass action. Moreover, we assume that the resting predator cells are converted to the hunting cells either by direct contact with them or by contact with a fast diffusing substance produced by hunting cells. We consider that once a cell has been converted, it will never return to the resting stage. Hence, active cells die at a constant probability per unit time.

7. Finally, we assume that during the resting phase, a predator and tumor cells are nutrient rich, undergoing mitosis, and the tumor cells have a proliferative advantage over the normal cells. If $\boldsymbol{x}(t), \boldsymbol{y}(t)$, and $\boldsymbol{z}(t)$ denote the densities of tumor cells, hunting predator cells, and resting predator cells at time $t$, respectively, the resulting dynamic system can be described by the following set of non-dimensional, nonlinear ordinary differential equations: 


$$
\begin{aligned}
& x^{\prime}=1+x\left(a_{1}(1-x)-y\right), \\
& y^{\prime}=y\left(a_{2} z-a_{3}\right), \\
& z^{\prime}=z\left(a_{4}(1-z)-a_{5} y-a_{6}\right) .
\end{aligned}
$$

\section{The Equilibria}

The equilibria are found by solving the system:

$$
\begin{aligned}
& 1+x\left(a_{1}(1-x)-y\right)=0 \\
& y\left(a_{2} z-a_{3}\right)=0 \\
& z\left(a_{4}(1-z)-a_{5} y-a_{6}\right)=0
\end{aligned}
$$

Therefore, this gives six equilibrium points as follows:

$$
\begin{aligned}
& E_{1}=\left[\frac{1}{2}\left(1+\sqrt{1+\frac{4}{a_{1}}}\right), 0,0\right], E_{2}=\left[\frac{1}{2}\left(1+\sqrt{1+\frac{4}{a_{1}}}\right), 0,\left(1-\frac{a_{6}}{a_{4}}\right)\right] \\
& E_{3}=\left[\frac { 1 } { 2 a _ { 1 } } \left(\left(a_{1}-x_{2}\right)\right.\right. \\
& \left.\left.\quad+\sqrt{\left(a_{1}-x_{2}\right)^{2}+4 a_{1}}\right),\left(\frac{a_{4}}{a_{5}}\left(1-\frac{a_{3}}{a_{2}}-\frac{a_{6}}{a_{4}}\right)\right), \frac{a_{3}}{a_{2}}\right] \\
& E_{4}=\left[\frac{1}{2}\left(1-\sqrt{1+\frac{4}{a_{1}}}\right), 0,0\right], \quad E_{5}=\left[\frac{1}{2}\left(1-\sqrt{1+\frac{4}{a_{1}}}\right), 0,\left(1-\frac{a_{6}}{a_{4}}\right)\right] \\
& E_{6}=\left[\frac { 1 } { 2 a _ { 1 } } \left(\left(a_{1}-x_{2}\right)\right.\right. \\
& \left.\left.\quad-\sqrt{\left(a_{1}-x_{2}\right)^{2}+4 a_{1}}\right),\left(\frac{a_{4}}{a_{5}}\left(1-\frac{a_{3}}{a_{2}}-\frac{a_{6}}{a_{4}}\right)\right), \frac{a_{3}}{a_{2}}\right]
\end{aligned}
$$

\section{Analytic Study of the Equilibria for the Biologically Feasible}

$E_{1}$ is biologically feasible.

$E_{2}$ is biologically feasible if $\quad 1-\frac{a_{6}}{a_{4}}>0$ or $a_{6}<a_{4} \ldots$

$E_{3}$ is biologically feasible if

$\frac{a_{4}}{a_{5}}\left(1-\frac{a_{3}}{a_{2}}\right)-\frac{a_{6}}{a_{5}} \geq 0$ or $1-\frac{a_{3}}{a_{2}}-\frac{a_{6}}{a_{4}} \geq 0$ and $\frac{a_{3}}{a_{2}}+\frac{a_{6}}{a_{4}} \leq 1$

However, we conclude from equations (1) \& (2) that $a_{3}<$ $a_{2} \& a_{6}<a_{4} \ldots$ (3)

Under these conditions, we discuss the stability of Equilibria $E_{1}, E_{2}$, and $E_{3}$

\section{The Eigenvalues of the Equilibria}

We will evaluate the eigenvalues of the three biological feasible critical points, and we note that these points are symmetric with the other equilibria.

\section{The Eigenvalues of $\boldsymbol{E}_{1}$}


$\lambda_{11}=-\sqrt{a_{1}^{2}+4 a_{1}}$
$\lambda_{12}=-a_{3}$
$\lambda_{13}=a_{4}-a_{6}$

The Egenvalues of $E_{2}$

$\lambda_{21}=-\sqrt{a_{1}^{2}+4 a_{1}}$

$\lambda_{22}=\left(a_{2} a_{4}-a_{3} a_{4}-a_{2} a_{6}\right) / a_{4}$,

$\lambda_{23}=a_{6}-a_{4}$

The Egenvalues of $\mathbf{E}_{\mathbf{3}}$

$\lambda_{31}=-k$

$\lambda_{32}=\left[\begin{array}{llll}-a_{3} a_{4}+\sqrt{a_{3}{ }^{2}} a_{4}{ }^{2}-4 a_{2}{ }^{2} & a_{3} & x_{2}\end{array}\right] / 2 a_{2}$

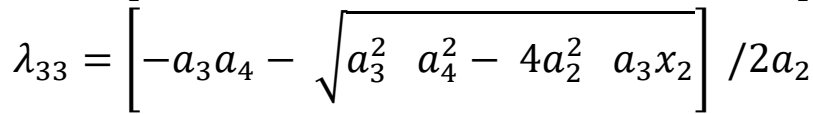

Where;

$k=\sqrt{\left(a_{1}-x_{2}\right)^{2}+4 a_{1}}$

$x_{2}=\left[a_{4}\left(a_{2}-\mathrm{a}_{3}\right)-a_{2} \mathrm{a}_{6}\right] / a_{2} a_{5}$

Stability Analyses

The Stability of $E_{1}$

The eigenvalues of $E_{1}$ are as follows:

$$
\lambda_{11}=-\sqrt{a_{1}{ }^{2}+4 a_{1}}, \quad \lambda_{12}=-a_{3}, \quad \lambda_{13}=a_{4}-a_{6}
$$

We see that the eigenvalue $\lambda_{13}$ is positive, so $E_{1}$ is unstable.

\section{The Stability of $E_{2}$}

The eigenvalues of $E_{2}$ are as follows:

$\lambda_{21}=-\sqrt{\mathrm{a}_{1}{ }^{2}+4 \mathrm{a}_{1}}, \lambda_{22}=\left(\mathrm{a}_{2} \mathrm{a}_{4}-\mathrm{a}_{3} \mathrm{a}_{4}-\mathrm{a}_{2} \mathrm{a}_{6}\right) / \mathrm{a}_{4}$ and

$\lambda_{23}=a_{6}-a_{4}$. We see that $\lambda_{21}<0$ and

$\lambda_{22}=\frac{\left(a_{2} a_{4}-a_{3} a_{4}-a_{2} a_{6}\right)}{\mathrm{a}_{4}}=\mathrm{a}_{2}\left(1-\frac{\mathrm{a}_{3}}{\mathrm{a}_{2}}-\frac{\mathrm{a}_{6}}{\mathrm{a}_{4}}\right)=a_{2}\left[1-\left(\frac{a_{3}}{a_{2}}+\frac{a_{6}}{a_{4}}\right)\right]>0$

$\lambda_{23}=a_{6}-a_{4}<0$, so $E_{2}$ is unstable.

\section{The Stability of $E_{3}$}

The eigenvalues of $E_{3}$ are as follows:

$\lambda_{31}=-k$, where $k=\sqrt{\left(a_{1}-x_{2}\right)^{2}+4 a_{1}}$,

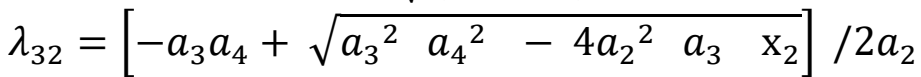

$\lambda_{33}=\left[-a_{3} a_{4}-\sqrt{a_{3}^{2} a_{4}^{2}-4 a_{2}^{2} a_{3} x_{2}}\right] / 2 a_{2}$ 
We observe that for $\lambda_{31}$, we see: $k>0$ and $\lambda_{31}$ is negative, and for $\lambda_{32}$ and $\lambda_{33}$, we observe that:

i) $\lambda_{32}$ and $\lambda_{33}$ are real and negative, if $a_{3} a_{4}^{2} \geq 4 a_{2}^{2} x_{2}$.

ii) $\lambda_{32}$ and $\lambda_{33}$ are complex with negative real parts, if $a_{3} a_{4}{ }^{2}<$ $4 a_{2}^{2} x_{2}$ or $a_{3} a_{4}<4 a_{2}^{2}\left(1-\frac{a_{3}}{a_{2}}-\frac{a_{6}}{a_{4}}\right)$.

Then, $E_{3}$ is always unstable.

\section{Time Series and Phase Plane}

Here, we give clear pictures of the behavior of the system, numerically, by drawing figures of time series and phase plane with different values of parameters and initial values:
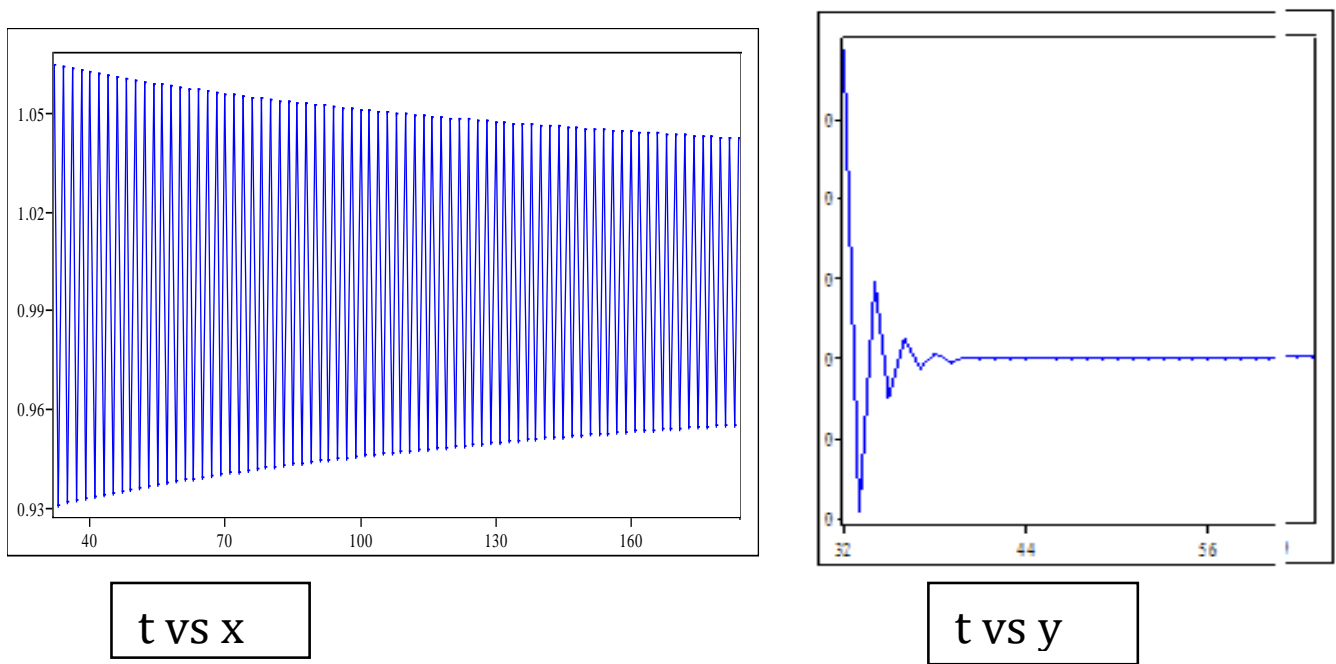

$$
\text { t vS } x
$$

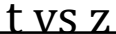

Figure 1a. Phase plane 1 )x vs y. 2)x vs z. 3)y vs z. a1 $=1.5$, a2=2, a3=1, a4=0.5, a5=0.1, a6 =0.25 initial values $(0.2,0.2,0.2)$ 
Figure 1b. Phase plane 1)x vs y. 2)x vs z. 3)y vs z. a1=1.5, a2=2, a3=1, a4=0.5, a5=0.1, a6=0.25 initial values $(0.2,0.2,0.2)$
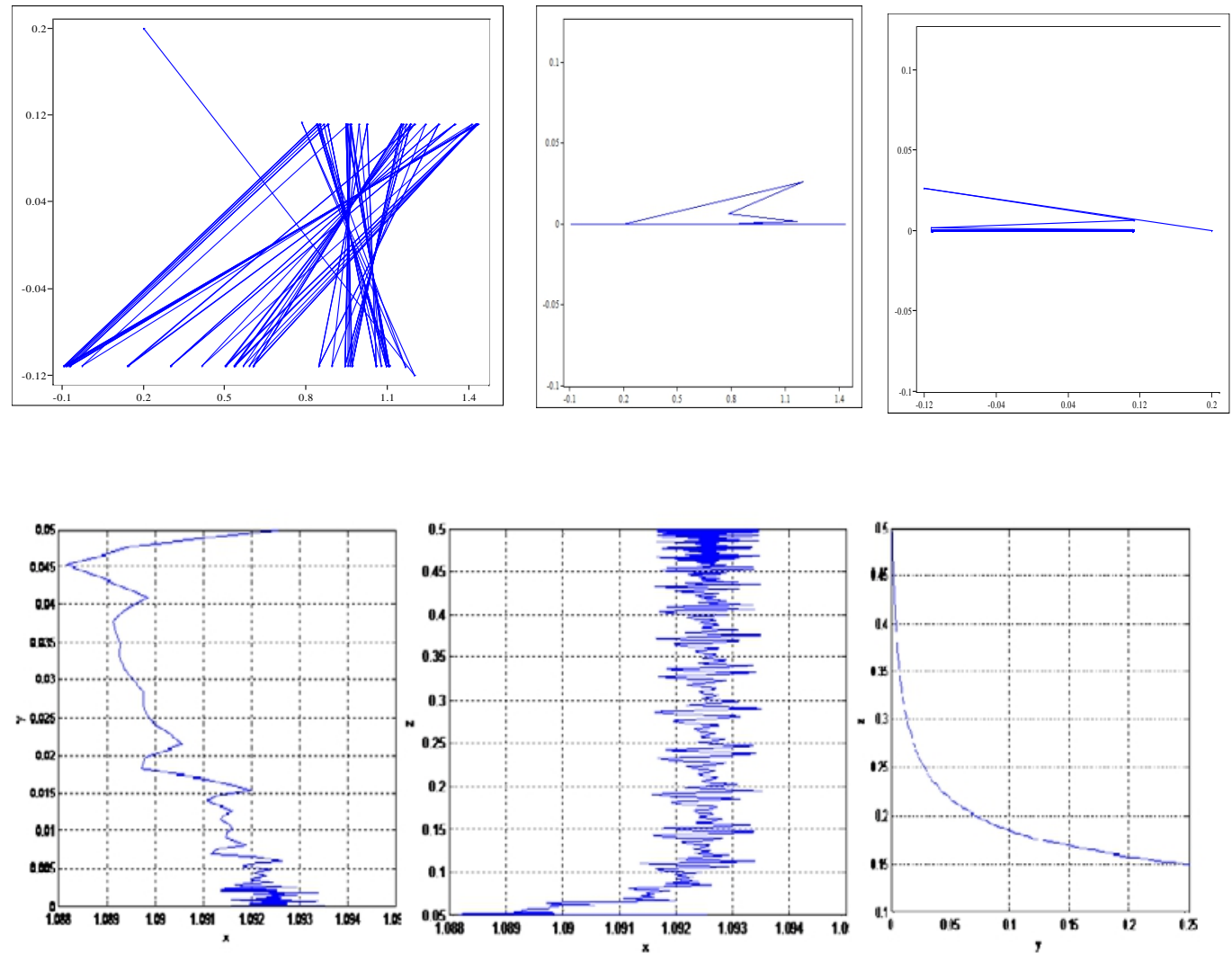

Figure 3: phase plane. 1)xvsy, 2)xvs $z, 3$ yvs $z$. a $1=9.89, a 2=2, a 3=1, a 4=0.5$, as $=0.1, a 6=0.25$, initial values (1.09255, 0.05, 0.05)
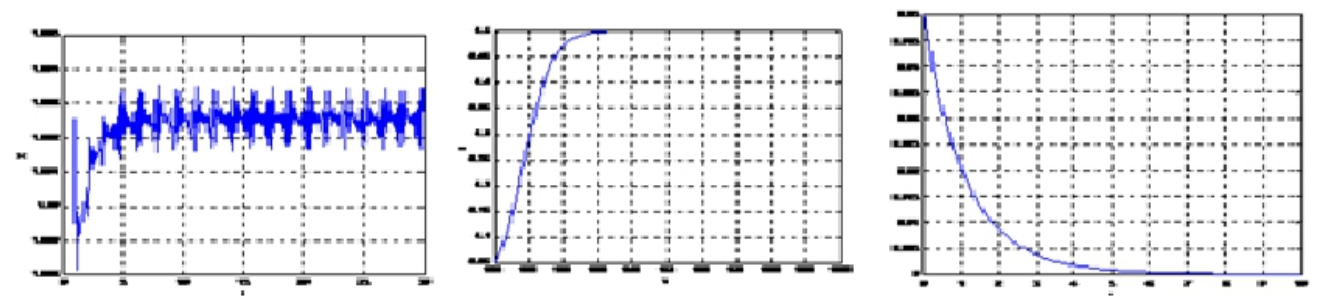

Figure 2: Time trace. 1)t vs $x, 2) t$ vs $y, 3) t$ vs $z$. $a 1=9.89, a 2=2, a 3=1, a 4=0.5$, a5 $=0.1, a 6=0.25$, initial values $(1.09255,0.05,0.05)$ 

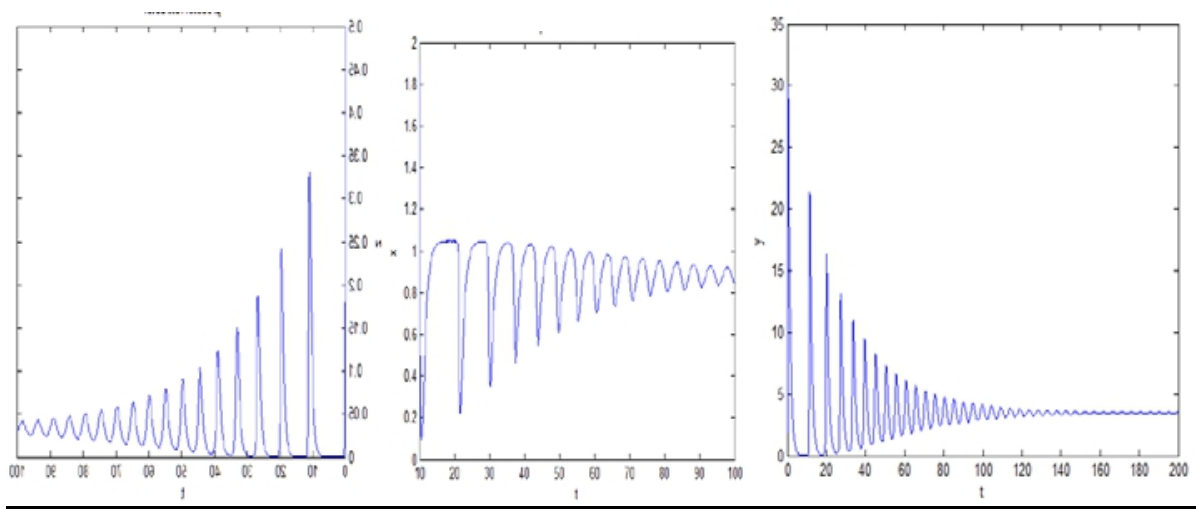

Figure 4. Time series, the parameter values: $a 1=20, a 2=30, a 3=1, a 4=2, a 5=0.5, a 6=0.2$, the initial values:

$(\mathrm{x} 0, \mathrm{y} 0, \mathrm{z} 0)=(0.05,0.2,0.2)$.
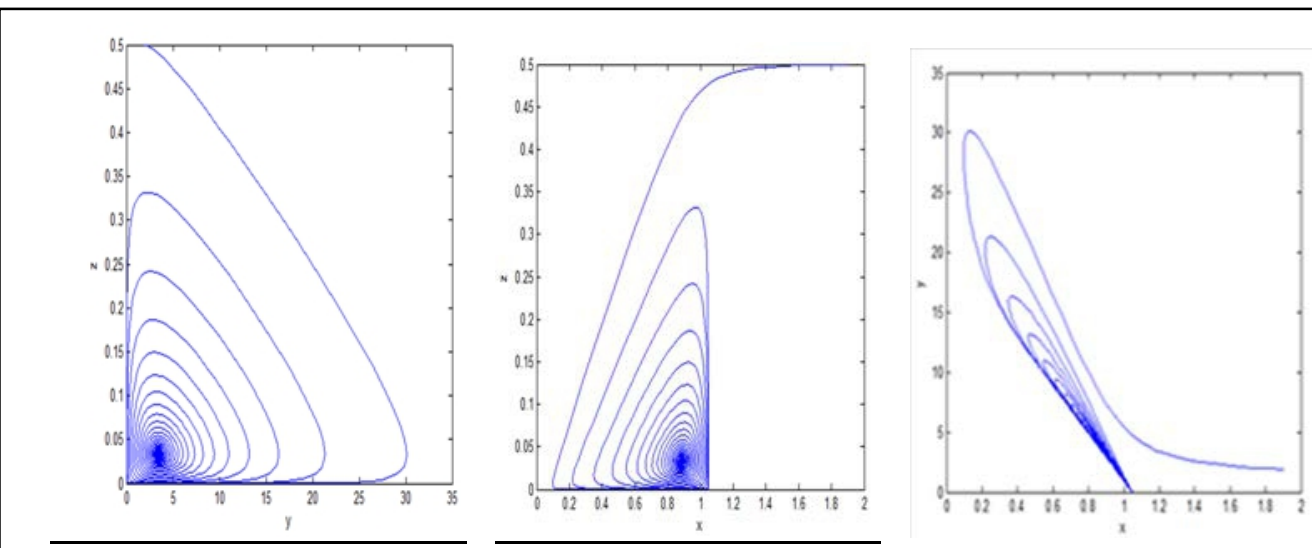

Figure 5 , phase plane, the parameter values: $a 1=20, a 2=30, a 3=1, a 4=2, a 5=0.5, a 6=0.2$, the initial values: $(\mathrm{x} 0, \mathrm{y} 0, \mathrm{z} 0)=(0.05,0.2,0.2)$.

\section{Bifurcation analysis}

We first see that $\mathrm{E}_{1}$ and $E_{2}$ are unstable with no cases under the conditions of the biologically feasibility.

But the third equilibrium $\mathrm{E}_{3}$ has many cases under the conditions of the biological feasibility: 
and negative i.e.,

1) if $a_{3} a_{4} \geq 4 a_{2}^{2}\left(1-\frac{a_{3}}{a_{2}}-\frac{a_{6}}{a_{4}}\right)$, then all the eigenvalues are real $\lambda_{31}<0, \lambda_{32}<0$ and $\lambda_{33}<0$

Thus, $\mathrm{E}_{3}$ is stable.

2) if $a_{3} a_{4}<4 a_{2}^{2}\left(1-\frac{a_{3}}{a_{2}}-\frac{a_{6}}{a_{4}}\right)$

Then, $\lambda_{31}<0$, and $\lambda_{32}$ and $\lambda_{33}$ are complex with a negative real part.

Thus, $\mathrm{E}_{3}$ is stable.

\section{Essential Concepts}

Variations in the control parameters of dynamic systems generate completely new, long-term patterns of motion. Qualitative changes in the topological texture of the trajectories in the phase space as a result of a change in one or more control parameters are called bifurcations and the appertaining critical parameters bifurcation values. For example, an original stable state of equilibrium can become unstable for a critical value, $\mu_{c r}$, of the control parameter and can give rise to two new stable states of equilibrium.

It is the purpose of a bifurcation analysis to determine the bifurcation value $\mu_{c r}$ and to construe the new bifurcating solutions for $\mu>\mu_{c r}$. A systemization of the bifurcating solutions is possible for one-dimensional systems with one control parameter. However, it is extremely difficult to develop higher-dimensional ones with several parameters. As a result, there is little research in this area (Argyris and Hase, 1994).

In this section, we will investigate the eigenvalues of the above three critical points $\mathrm{E}_{1}, \mathrm{E}_{2}$, and $\mathrm{E}_{3}$, since the other three critical points are symmetric with these points.

\section{Bifurcation Cases}

Using the concepts of bifurcation, degenerate critical points, and bifurcation parameter values, some analytical investigation given include many cases with numerical aspects:

\section{i) Case 1}

When $a_{4}=a_{6}$, we see that:

$\lambda_{13}=a_{4}-a_{6}=0$, and $\mathrm{E}_{1}$ has a zero eigenvalue and is a bifurcation point where a bifurcation arises. At this value of parameters also, $\lambda_{23}=a_{6}-a_{4}=$ 0 , and $E_{2}$ is a bifurcation point which gives a special phenomenon that is two bifurcation points with the same bifurcation value. We observe that $E_{2}$ merges with $E_{1}$ and becomes the same, i.e. 
$E_{2}=\left[\frac{1}{2}\left(1+\sqrt{1+\frac{4}{a_{1}}}\right), 0,1-\frac{a_{6}}{a_{4}}\right]$ changes $\quad$ to $\quad$ become $\quad \mathrm{E}_{1}=$ $\left[\frac{1}{2}\left(1+\sqrt{1+\frac{4}{a_{1}}}\right), 0,0\right]$. Also, $\mathrm{E}_{5}$ merges with $\mathrm{E}_{4}$ i.e $\mathrm{E}_{5}$ changes to become $E_{4}=\left[\frac{1}{2}\left(1-\sqrt{1+\frac{4}{a_{1}}}\right), 0,0\right], \quad$ and $E_{3}$ changes to become non biologically feasible. Since $\mathrm{y}_{3}=-\frac{1}{a_{5}} \frac{a_{3}}{a_{2}}<0$, then the total number of equilibria changes from 6 to 4 . Thus, the case $a_{4}=a_{6}$ is a bifurcation case. Numerically, when fixed values of parameters satisfy the biological feasibility, we verify the situation above. First, taking the values with $\mathrm{a}_{6} \neq \mathrm{a}_{4}: \mathrm{a} 1=0.4, \mathrm{a} 2=9.9, \mathrm{a} 3=0.1, \mathrm{a} 4=5.6, \mathrm{a} 5=4.6, \mathrm{a} 6=0.5$, gives the following critical points $\mathrm{E}_{1}=(2.1583,0,0), \mathrm{E}_{2}=(2.1583,0,9179)$ $\mathrm{E}_{3}=(0.9344,1.0964,0.0101), \quad \mathrm{E}_{4}=(-1.1583,0,0)$, $E_{5}=(-1.1583,0,9179)$, and $E_{6}=(-2.6754,1.0964,0.0101)$, that is six points. And calculating the eigenvalues of the three first critical points $E_{1}$, $E_{2}$, and $E_{3}$ gives the following: For $E_{1}, \lambda_{11}=-0.1, \lambda_{12}=-1.3266$, and $\lambda_{13}=5.1$, for $E_{2}, \lambda_{21}=-5.1, \lambda_{22}=8.9161$, and $\lambda_{23}=-1.3266$, and for $\mathrm{E}_{3}, \lambda_{21}=-1.4439, \lambda_{22}=-0.0283+0.7096 \mathrm{i}$, and $\lambda_{23}=-0.0283-$ $0.7096 \mathrm{i}$.

Second, taking the values with $a_{4}=a_{6}: \mathrm{a} 1=2$, a2 $=0.5, \mathrm{a} 3=0.4$, $\mathrm{a} 4=0.5, \mathrm{a} 5=0.8, \mathrm{a} 6=0.5$ gives the following critical points: $\mathrm{E}_{1}=$ $(1.3660,0,0), E_{2}=(-0.3660,0,0), \quad E_{3}=(1.5687,-0.5,0.8), \quad$ and $\mathrm{E}_{4}=(-0.3187,-0.5,0.8)$, that reduce to four points. And calculating the eigenvalues of the first critical point, $\mathrm{E}_{1}$ in this case, gives

$$
\lambda_{11}=-0.4, \quad \lambda_{12}=0, \quad \lambda_{13}=-3.4641 \text {, }
$$

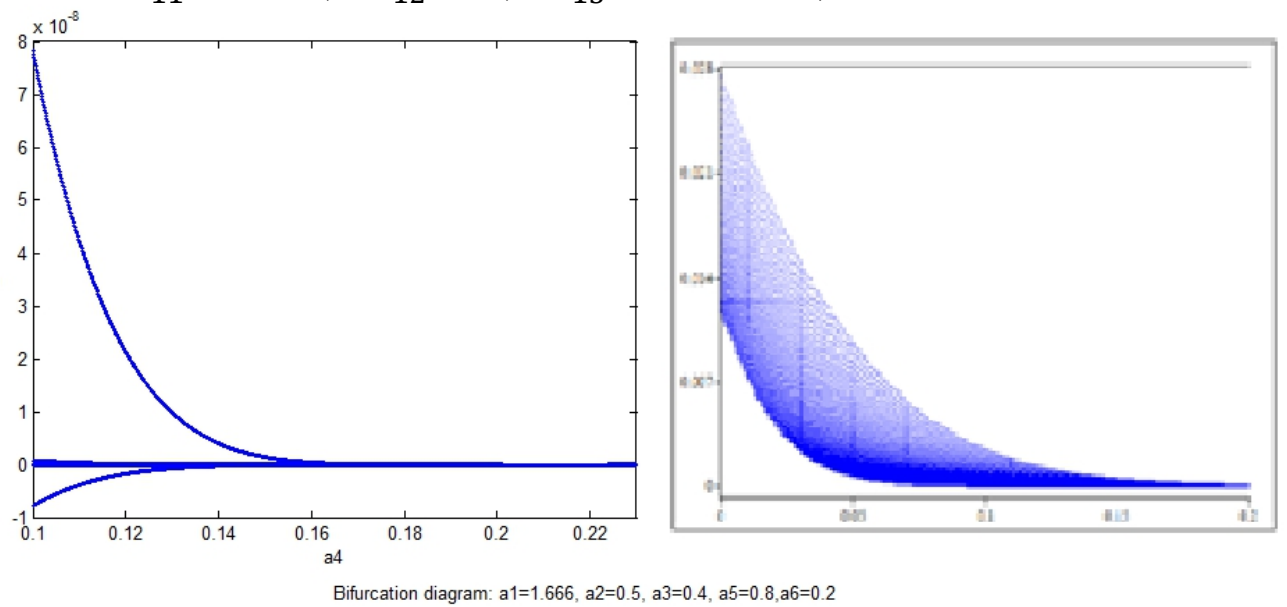

Figure 6. Bifurcation Diagram of Case 1 


\section{ii) Case 2}

When $\frac{a_{3}}{a_{2}}+\frac{a_{6}}{a_{4}}=1$, we observe that $\frac{\mathrm{a}_{6}}{\mathrm{a}_{4}}=1-\frac{\mathrm{a}_{3}}{\mathrm{a}_{2}}$ and $x_{2}=0$, which gives $\lambda_{22}=0$. Thus, $E_{2}$ has a zero eigenvalue, and we observe that $\lambda_{32}=0$ and $E_{3}$ has also a zero eigenvalue in the same time.

And then $E_{3}$ merges with $E_{2}$, and $E_{6}$ merges with $E_{5}$. Thus, the number of equilibria changes from 6 to 4 . Numerically, as in case 1, when the fixed values of parameters satisfy the biological feasibility, we verify the situation above. First, taking the values with $a_{6} \neq a_{4}\left(1-\frac{a_{3}}{a_{2}}\right): a 1=0.4$, $\mathrm{a} 2=9.9, \mathrm{a} 3=0.1, \mathrm{a} 4=5.6, \mathrm{a} 5=4.6, \mathrm{a} 6=0.5$, gives the following critical points $\mathrm{E}_{1}=(2.1583,0,0)$ $\mathrm{E}_{2}=(2.1583,0,9179)$, $\mathrm{E}_{3}=(0.9344,1.0964,0.0101)$, $\mathrm{E}_{4}=(-1.1583,0,0)$, $E_{5}=(-1.1583,0,9179)$, and $E_{6}=(-2.6754,1.0964,0.0101)$, that is six points. And calculating the eigenvalues of the first three critical points $E_{1}$, $E_{2}$, and $E_{3}$ gives the following: For $\mathrm{E}_{1}, \lambda_{11}=-0.1, \lambda_{12}=-1.3266$, and $\lambda_{13}=5.1$, for $\mathrm{E}_{2}, \lambda_{21}=-5.1, \lambda_{22}=$ 8.9161, and $\lambda_{23}=-1.3266$, and for $\mathrm{E}_{3}, \lambda_{31}=-1.4439, \lambda_{32}=-0.0283+$ $0.7096 \mathrm{i}$, and $\lambda_{33}=-0.0283-0.7096 \mathrm{i}$.

Second, taking the values with $\mathrm{a}_{6}=\mathrm{a}_{4}\left(1-\frac{\mathrm{a}_{3}}{\mathrm{a}_{2}}\right): \mathrm{a} 1=2$, $\mathrm{a} 2=0.5$, $\mathrm{a} 3=0.4, \mathrm{a} 4=0.5, \mathrm{a} 5=0.8, \mathrm{a} 6=0.1$ gives the following critical point $\mathrm{E}_{1}=$ $(1.3660,0,0), \quad E_{2}=(0.3660,0,0), \quad E_{3}=(1.3660,0,0.8), \quad$ and $E_{4}=$ $(-0.3660,0,0.8)$, that reduce to four points. And calculating the eigenvalues of the third critical point, $E_{3}$, gives the following: $\lambda_{31}=-0.4$, $\lambda_{32}=0$, and $\lambda_{33}=-3.4641$.

Here, $\mathrm{a}_{6}=\mathrm{a}_{4}\left(1-\frac{\mathrm{a}_{3}}{\mathrm{a}_{2}}\right)$ leads to taking $\mathrm{a}_{6}$ as a bifurcation parameter. We draw the bifurcation diagram in this case by using fixed values of the other parameters as shown in figure 7 below.

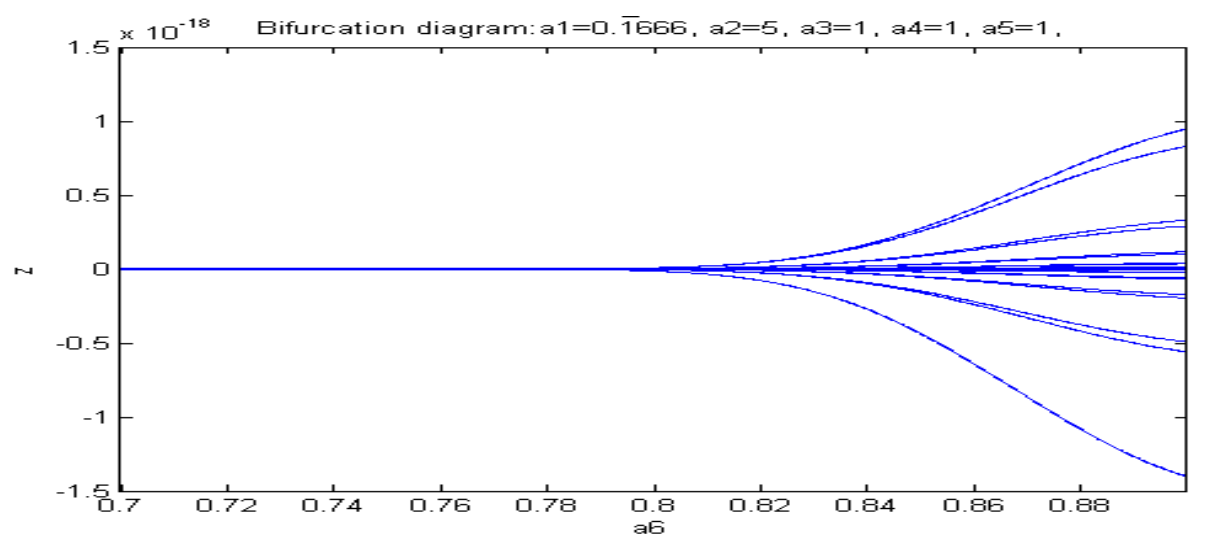

Figure 7. Bifurcation Diagram of Case 2 


\section{iii) Case 3: Composition of Cases}

This is a complicated case since many elements relate together in the eigenvalue $\quad \lambda_{32}=\left[\begin{array}{llll}-a_{3} a_{4}+\sqrt{a_{3}^{2}} a_{4}^{2}-4 a_{2}^{2} & a_{3} & x_{2}\end{array}\right] / 2 a_{2}$ which gives different influences as follows:

1- When $a_{4}=a_{6}$, then $\mathrm{x}_{2}<0$, which leads $\lambda_{32}$ to becoming positive, and the critical point $\mathrm{E}_{3}$ becoming an unstable saddle point.

2- When $\frac{a_{3}}{a_{2}}+\frac{a_{6}}{a_{4}}=1$, then $\mathrm{x}_{2}=0$, which leads $\lambda_{32}$ to becoming zero, and the critical point $\mathrm{E}_{3}$ becoming a bifurcation point.

3- When $\frac{a_{3}}{a_{2}}+\frac{a_{6}}{a_{4}}<1$, then $\mathrm{x}_{2}>0$ and $a_{3} a_{4} \geq 4 a_{2}^{2}\left(1-\frac{a_{3}}{a_{2}}-\frac{a_{6}}{a_{4}}\right)$ lead $\lambda_{32}$ to becoming real and negative, and the critical point $E_{3}$ becoming a stable node point.

4- When $\frac{a_{3}}{a_{2}}+\frac{a_{6}}{a_{4}}<1$, then $\mathrm{x}_{2}>0$ and $a_{3} a_{4}<4{a_{2}}^{2}\left(1-\frac{a_{3}}{a_{2}}-\frac{a_{6}}{a_{4}}\right)$ lead $\lambda_{32}$ to becoming complex with negative real parts, and the critical point $\mathrm{E}_{3}$ becoming a stable focus point.

These different elements give good pictures of the bifurcation phenomena, using different values of the arbitrary parameters and initial values as the following figure shows.

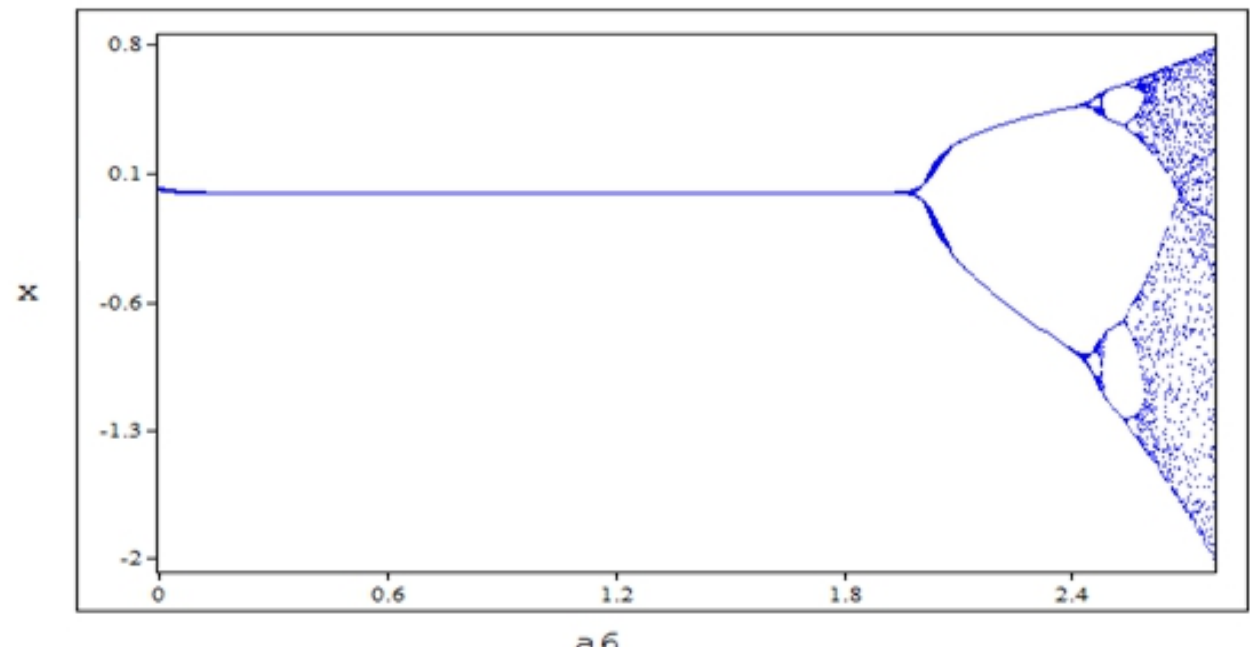

Figure 8. Bifurcation Diagram. The parameter values: $a 1=0.16666, a 2=2, a 3=1, a 4=1$, $\mathrm{a} 5=2$ and $\mathrm{a} 6$ is the bifurcation parameter. The initial values: $(\mathrm{x} 0, \mathrm{y} 0, \mathrm{z} 0)=(3.1,0.1,0.6)$. 
And then, we give clear pictures of some sub regions of the above diagrams by enlarging some of its parts as follows:
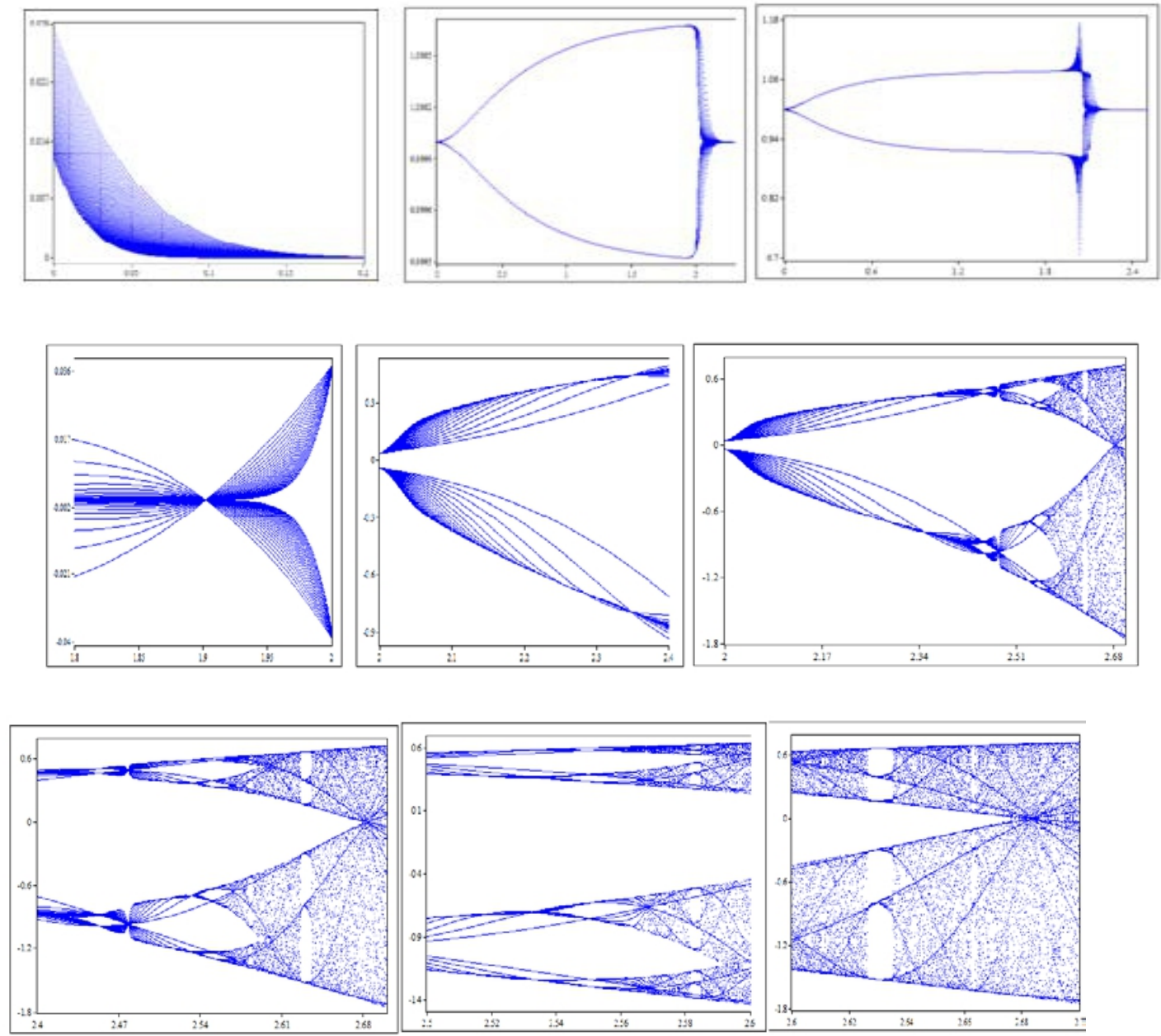

\section{Other Bifurcation Diagrams}

In the following cases, we get other bifurcation diagrams arising at certain values of the system parameters which are different from the above cases, as shown in the following two figures. 

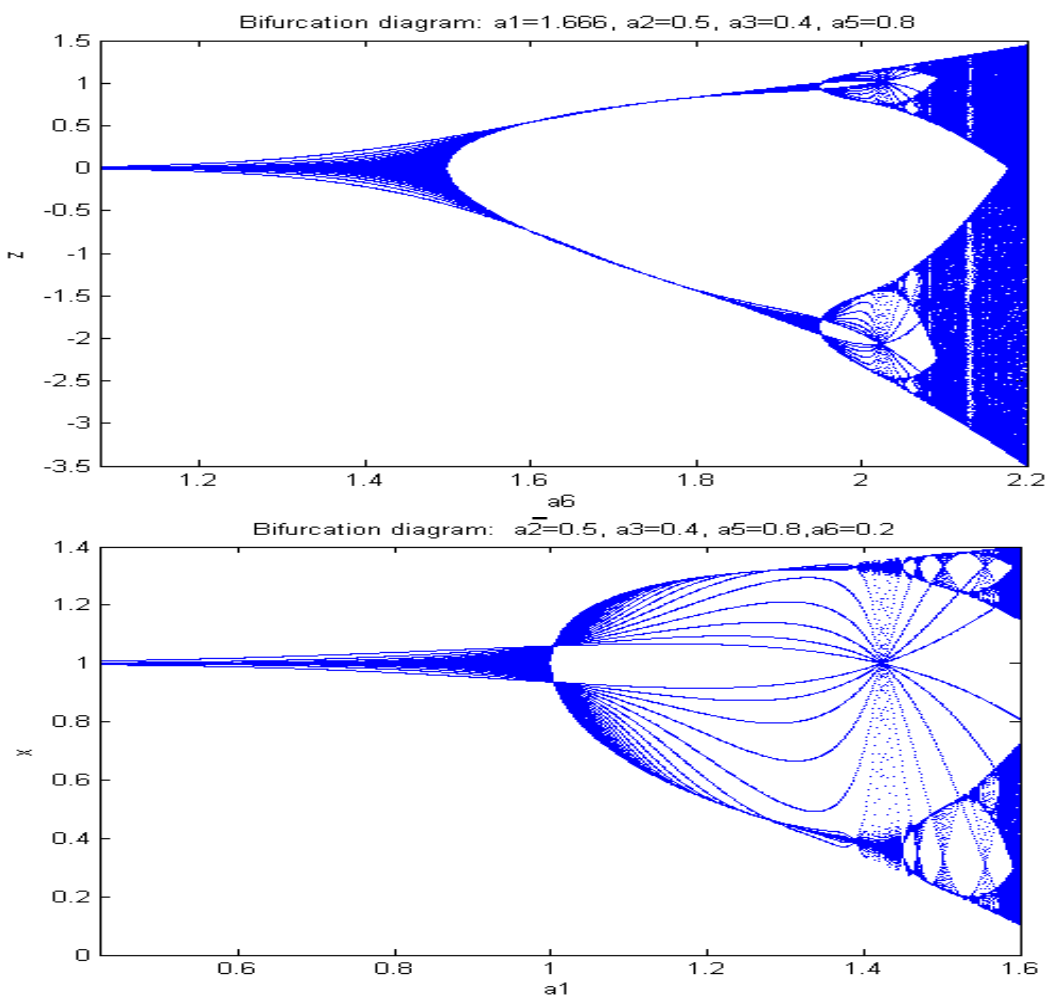

Here, we get another case that is a strange picture of a bifurcation diagram where two bifurcation phenomena arise in the same range of the bifurcation parameter a1 with certain values of the other parameters. The two phenomena also begin in the negative area of the bifurcation parameter a1 and then disappear in that range. Then, in the positive area of the bifurcation parameter a1, the two phenomena begin again with a big range of the variable $\mathrm{x}$.

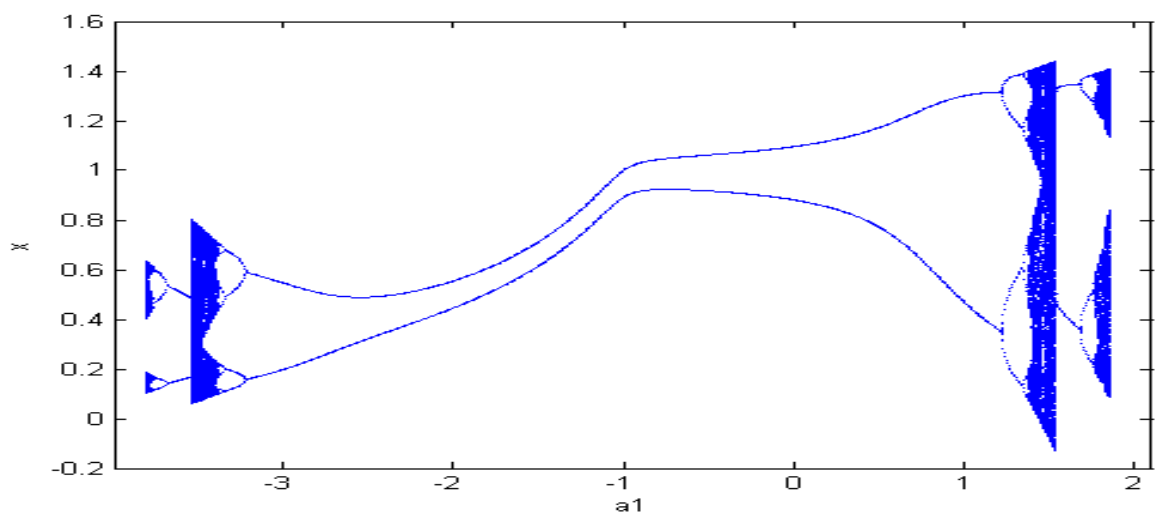

Figure 9. Bifurcation diagram. The parameter values: a2=2, a3=1, a4=0.5, $\mathrm{a} 5=0.1, \mathrm{a} 6=0.21$ and $\mathrm{a} 1$ is the bifurcation parameter. The initial values: $(\mathrm{x} 0, \mathrm{y} 0$, 


\section{Conclusion}

In this paper, we analytically and numerically studied a mathematical biological system model given in Gohary and Alwasel (2009) for stability and bifurcation. We gave an investigation into the equilibrium points for the biological feasibility, stability, phase planes, and time trace for the behavior of the system near those points. The bifurcation analytical study determined (recognized) many cases where bifurcation held, which was verified using examples with fixed values for each case. Finally, we drew many bifurcation diagrams for those cases which showed us that the system has rich behaviors with bifurcation phenomena.

\section{References:}

Bellomo, Li, \& Maini (2008). On the foundations of cancer modelling: selected topics, speculations, and perspectives. Mathematical Models \& Methods in Applied Sciences, vol. 18, no. 4, pp. 593-646

Bellomo, Bellouquid, Nieto, \& Soler (2010). Multiscale biological tissue models and flux-limited chemotaxis for multicellular growing systems. Mathematical Models \& Methods in Applied Sciences, vol. 20, no. 7, pp. 1179-1207.

Chaplain, L., W. Gu, \& A. Radunskaya (2006). Mixed immunotherapy and chemotherapy of tumors: modeling, applications and biological interpretations. Journal of Theoretical Biology, pp.238-243, 841.

El-Gohary \& Alwasel (2009). The chaos and optimal control of cancer model with complete unknown parameters. Chaos, Solitons and Fractals 42, 2865-2874

Fateme Ghomanjani (2012). Bezier Control Points Method to Solve Scheduling of Injections of Immunotherapeutic Agents. Intelligent Control and Automation, 3, 20-25 doi:10.4236/ica.2012.31003 Published Online February 2012 (http://www.SciRP.org/journal/ica)

Francesco Pappalardo, Ferdinando Chiacchio \& Santo Motta (2013). Review Article Cancer Vaccines: State of the Art of the Computational Modeling Approaches. Hindawi Publishing Corporation, BioMed Research International, Article ID 106407, 6 pages, http://dx.doi.org/10.1155/2013/106407.

Frank \& Freedman (2000). A mathematical model of cancer treatment by

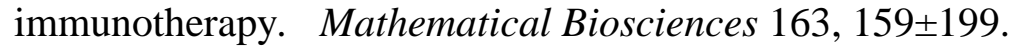

Gantmacher (1959). Applications of the Theory of Matrices. New York: Wiley, pp. 230.

Gilboa (2004). The promise of cancer vaccines. Nature Reviews Cancer, vol. 4, no. 5, pp. 401-411. 
John Argyris \& Maria Hase (1994). An Exploration of Chaos: An Introduction for Natural Scientists and Engineers. NORTH-HOLLAND.

Lisette de Pillis, K. Renee Fister, Weiqing Gu, Craig Collins, Michael Daub, David Gross, James Moore \& Benjamin Preskill (2009). Mathematical model creation for cancer chemo-immunotherapy1. Computational and Mathematical Methods in Medicine. Vol. 10, No. 3, 165-184.

Lisette de Pillis, Gu, \& Radunskaya (2006). Mixed immunotherapy and chemotherapy of tumors: modeling, applications and biological interpretations. Journal of Theoretical Biology. pp. 238-241, 4.

Rajib Mazumder \& Alam (2012). Design and Analysis of Chemotherapy and Immunotherapy Drug Scheduling Model for Cancer Tumour Treatment. Dhaka Univ. J. Sci. 60(2): 231-237.

Rihan, Safan, Abdeen, \& Abdel Rahman (2012). Qualitative and Computational Analysis of a Mathematical Model for Tumor-Immune Interactions. Hindawi Publishing Corporation, Journal of Applied Mathematics, Article ID 475720, 19 pages doi:10.1155/2012/475720.

W. Zou (2005). Immunosuppressive networks in the tumour environment and their therapeutic relevance. Nature Reviews Cancer, vol. 5, no. 4, pp. 263-274, 2005.

Y.Takeuchi, Yasuhiro, Iwasa, Yoh, Sato, Kazunori (Eds.). (2007). Mathematics for Life Science and Medicine. Bill Satzer, MathDL, http://www.springer.com/978-3-540-34425-4.

Zvia Agur \& Stanimir Vuk-Pavlović (2012). Personalizing immunotherapy Balancing predictability and precision. OncoImmunology 1:7, 1169-1171; (C) 2012 Landes Bioscience. 